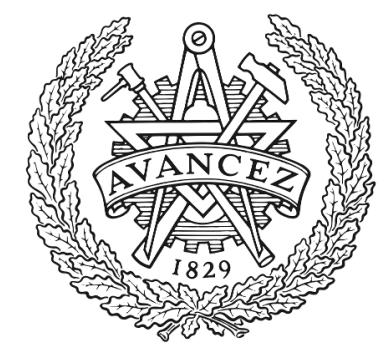

CHALMERS

UNIVERSITY OF TECHNOLOGY

\title{
Manufacturing full density powder metallurgy gears through HIP:ing
}

Downloaded from: https://research.chalmers.se, 2023-04-26 12:57 UTC

Citation for the original published paper (version of record):

Andersson, M., Bergendahl, M., Bjarre, U. et al (2019). Manufacturing full density powder metallurgy gears through HIP:ing. Metal Powder Report, 74(4): 199-203.

http://dx.doi.org/10.1016/j.mprp.2018.12.076

N.B. When citing this work, cite the original published paper. 


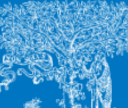

\title{
Manufacturing full density powder metallurgy gears through HIP:ing
}

\section{Michael Andersson ${ }^{a{ }^{*}}$, Magnus Bergendahl ${ }^{b}$, Ulf Bjarre ${ }^{c}$, Anders Eklund ${ }^{d}$, Staffan Gunnarsson ${ }^{e}$, Sven Haglund ${ }^{f}$, Hans Hansson ${ }^{g}$, Irma Heikkilä ${ }^{f}$, Alireza Khodaee ${ }^{\text {h }}$, Arne Melander ${ }^{i}$, Harald Nyberg ${ }^{c}$, Lars Nyborg ${ }^{j}$, Annika Strondl $^{f}$, Maheswaran Vattur Sundaram}

\author{
${ }^{a}$ Höganäs $A B$, Sweden \\ ${ }^{b}$ Volvo $A B$, Sweden

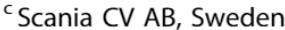 \\ ${ }^{d}$ Quintus Technologies $A B$, Sweden \\ e Uddeholm AB, Sweden \\ ${ }^{f}$ Swerim $A B$, Sweden \\ ${ }^{9}$ Swepart Transmission AB, Sweden \\ ${ }^{\mathrm{h}}$ KTH Royal Institute of Technology, Sweden \\ Swerea KIMAB, Sweden \\ ${ }^{\mathrm{j}}$ Chalmers University of Technology, Sweden
}

\section{Introduction}

In many cases powder metallurgy (PM) offers the most cost efficient way to make components on a larger scale, for instance in automotive applications. Complex shapes can be compacted near net shape and reduce the overall number of process steps required for manufacturing. Other benefits of PM include the elimination of scrap, where up to $98 \%$ of the raw material goes into the final component, and the possibility to tailor material compositions for specific applications.

A drawback of the PM technology is the inherent $5-15 \%$ of porosity in the components, which decreases the strength of the material compared to full density wrought steels. Techniques such as powder forging or rolling densification can be used to reduce or remove porosity in various components to improve strength. It is also possible to use hot isostatic pressing (HIP) to make fully dense parts from powder. A previous investigation, see for instance MPR Volume 72, number 2, 2017, demonstrated for instance that a HIP:ing a PM steel reduced the wear of a dog clutch of a rally car compared to the standard steel mating part.

\footnotetext{
* Corresponding author.

E-mail address: Andersson, M. (michael.andersson@hoganas.com)
}

However, HIP:ing normally requires the powder to be included in a canister during the process, which is normally a very expensive process.

The idea, is therefore, to combine pressing and sintering of conventional PM technology with a subsequent HIP:ing step to remove the remaining porosity. If the part is made to a sufficiently high density before the HIP step to have closed porosity the canister is not necessary, thus combining the advantages of conventional PM and HIP.

This paper summarizes the project HIPGEAR, which was run as a part of the Swedish FFI program between 2014 and 2018, and included a number of partners from industry and academia. The purpose of the project was to develop the can-less HIP process. The ultimate goal was to manufacture and test a demonstrator gear looking at applications for trucks.

\section{The process}

The main requirement for reaching full density after HIP:ing is to start with a component that has closed porosity to prevent the penetration of the process media into the material. In practice this means reaching at least around $7.4 \mathrm{~g} / \mathrm{cm}^{3}$ around the surface of the material. Figure 1 shows the difference of a gear tooth 

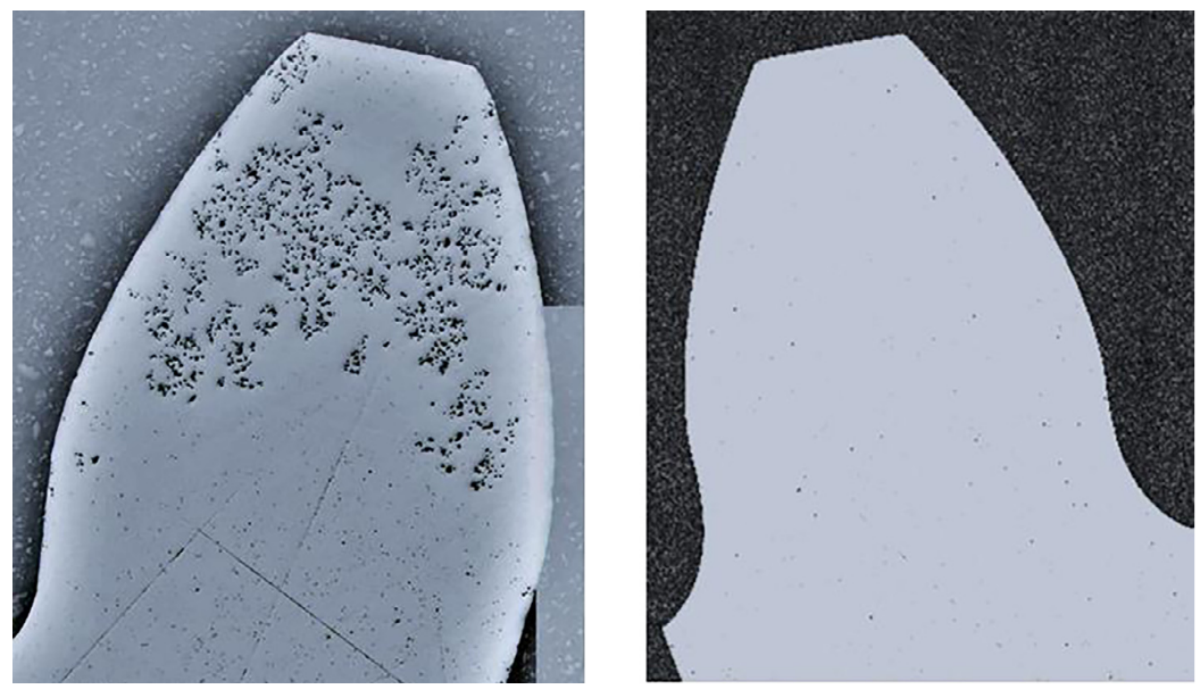

\section{FIGURE 1}

Illustration of HIP:ing with a. still open porosity and b. closed porosity.

where the density was too low versus another example with sufficient density to densify throughout.

Early on in this project, it was decided to use double pressing to reach sufficient density for successful HIP:ing. A previous study, see [1], demonstrated that it is possible to use a single compaction step to reach sufficient density. However, since that concept included finer than standard powders in combination with lower than normal lubricant content and high compaction pressures, it was decided not to use that process for the demonstrator in this project. With new powder mix concepts it is however believed that a single compaction solution will be possible.

Another question that had to be addressed was surface porosity. Even if the bulk density is sufficient to close the porosity pores that are open to the surface cannot close during HIP:ing. This is for instance seen in Figure 2, where some porosity still exists down to a depth of around $200 \mu \mathrm{m}$ even though full density was reached below.

Initially, different powder mix concepts were evaluated, and also the option to use finer powders to facilitate the closing of the porosity. However, in the end it was decided to use a standard powder grade, Astaloy ${ }^{\mathrm{TM}}$ Mo from Höganäs Ab, which is a pre alloyed iron powder with $1.5 \%$ Mo. The type of powder mix used was IntralubeE ${ }^{\circledR}$, with $0.3 \%$ C-F10 added.

Also, the final sintering step can be used to close the porosity. But in order to enhance that effect a high sintering temperature is typically necessary. In the end it was decided to sinter the material at $1300{ }^{\circ} \mathrm{C}$ for $60-90 \mathrm{~min}$. For HIP:ing a standard cycle

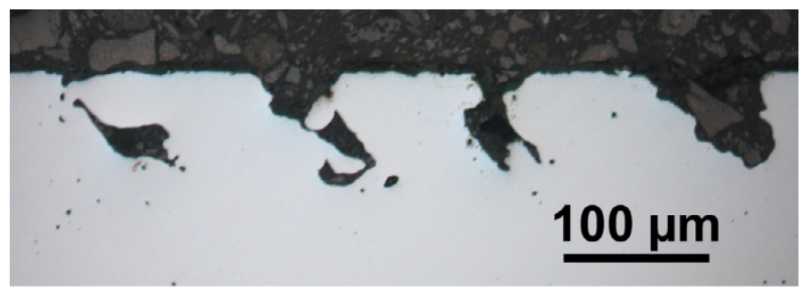

FIGURE 2

Open pores close to the surface. was used with 1000 bars of pressure at $1150^{\circ} \mathrm{C}$ for $120 \mathrm{~min}$ using a QIH21 HIP from Quintus Technologies.

It was found that a big drawback with the prolonged exposure to high temperatures, both at the final sintering step and during HIP:ing was extensive grain growth. Figure 3 shows average grain size after second compaction, final sintering and after HIP compared to a reference gear steel. From the picture, it is clear that both sintering and HIP:ing grows the grains significantly. Later on, it was also found that the larger grains decreased the fatigue strength, see also below.

To reduce the grain size again it was decided to do a normalization after HIP:ing. This was done by heating up the specimens to $920^{\circ} \mathrm{C}$ and with a hold times of either 2 or $30 \mathrm{~min}$ and then quench in water. With this process step, it was then possible to obtain a material with grain size comparable to the reference. In this case it's possible to do the normalization inside the HIP, thus avoiding any additional process steps.

\section{Simulations}

One part of the project focused on simulations as a tool for process development, where especially the compaction and HIP steps were modeled. By using advanced finite element simulations it's possible to simulate the density distributions after the different steps. Especially during HIP:ing there will be large dimensional changes, and the presence of density gradients in the component can then cause distortions. Here simulations can be used to estimate the distortions and compensate for them in the tool and process design. Further details can be found in [2].

Different material models were adapted and calibrated to compaction curves in order to describe the powder compact at different stages of the process. The first compaction, where the green body was formed from powder, was modeled using the CAP model. For the subsequent steps, the Gurson models were used to describe the deformation of a porous solid body. For the compaction simulations it's also important to include for instance the friction between powder and die to be able to capture for instance the neutral zone. Figure 4 shows an example 


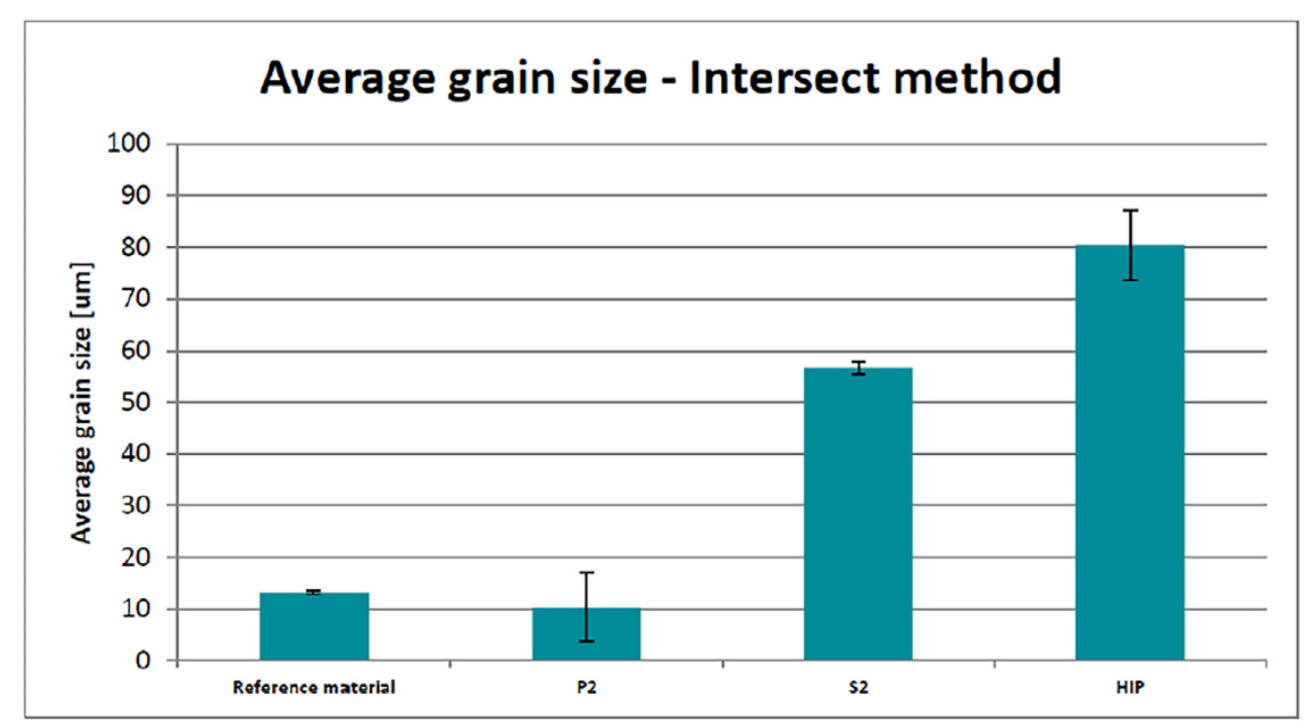

\section{FIGURE 3}

Grain size at different process steps.

of how a simulated relative density distribution looks after compaction.

Another area where the simulations proved useful is to compare how suitable different gear geometries are for the PM/HIP process. By looking at different gear data and simulating the minimum relative density it's possible to judge for instance the risk of residual porosity after HIP:ing.

\section{Demonstrator}

A large part of the project focused on developing a demonstrator gear in order to show that the process can be used to manufacture an actual component. Different options for the demonstrator were discussed early on in the project. Since the largest press available for the demonstrator was 800-tons, and since the process requires high compaction pressures it proved difficult to find a suitable truck component that could be used due to the typical size of these components. Ideally, it should also be possi- ble to use the part for fatigue testing to benchmark the performance of the HIP:ed component.

In the end, it was decided to use the C-PT type gears normally used for FZG testing, see Figure 5. A big advantage of this gear is that it can easily be used for fatigue testing, both tooth root bending fatigue in a pulsator, and contact fatigue in an FZG test rig. Also, even though the intended applications to a large extent are helical gears, it was decided that it would be better to start with a spur gear to learn more about how the shape and distortions develop during the process.

It should be noted that for the demonstrator a special compaction tooling was acquired for double pressing of a component with the right dimensions to accommodate for the HIP process.

The process to manufacture a gear was (parameters within parenthesis):

1. first compaction $(800 \mathrm{MPa})$

2. pre sintering $\left(800^{\circ} \mathrm{C}, 30 \mathrm{~min}\right)$
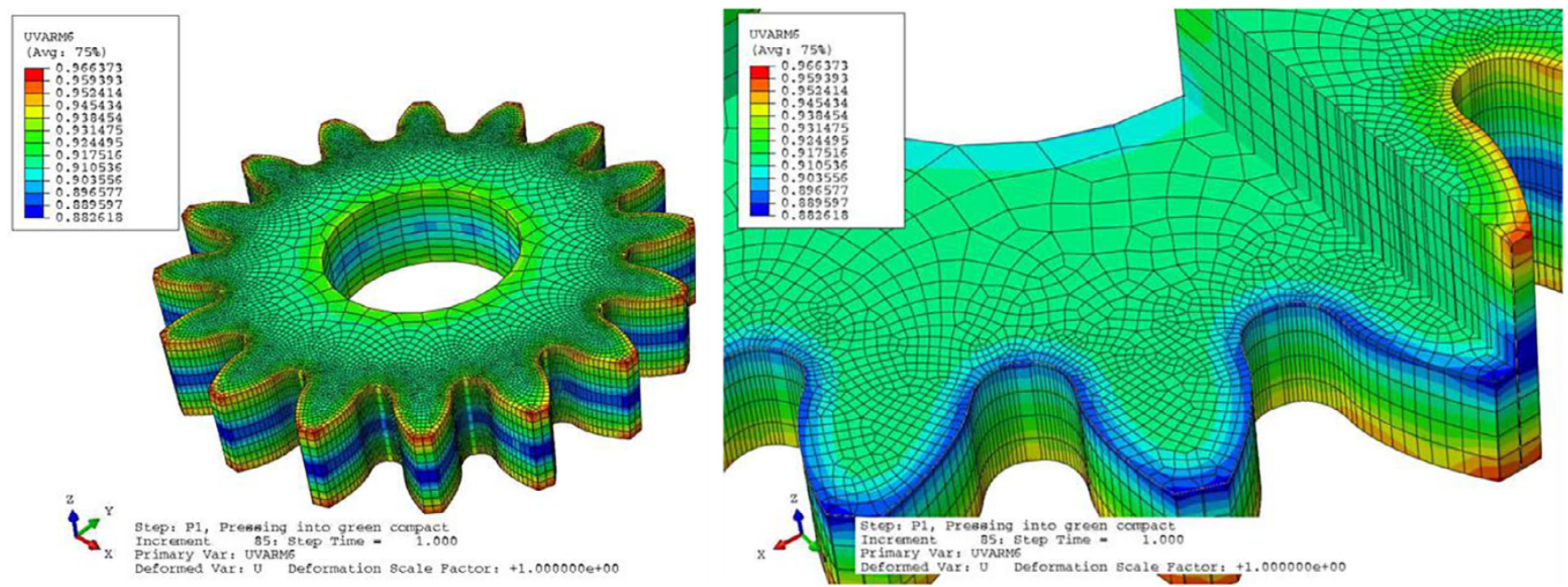


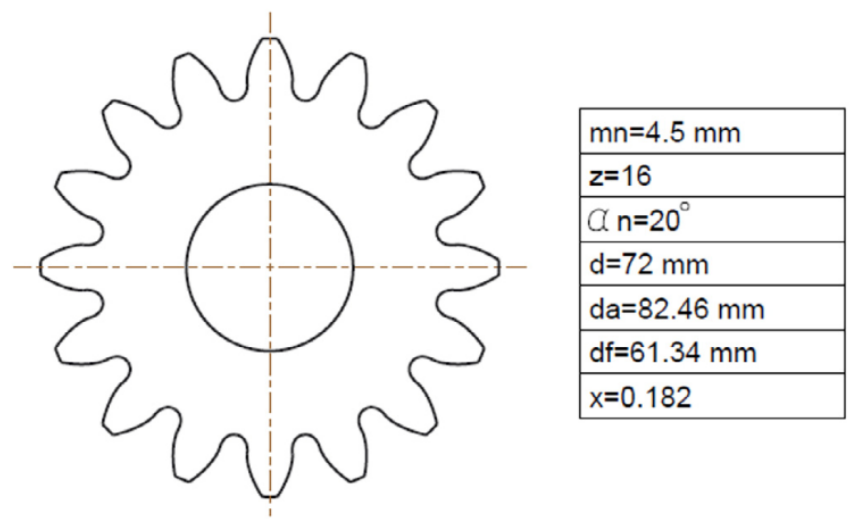

FIGURE 5

The C-PT gear geometry.

3. second compaction $(850 \mathrm{MPa})$

4. final sintering $\left(1300^{\circ} \mathrm{C}, 90 \mathrm{~min}\right)$

5. HIP $\left(1150^{\circ} \mathrm{C}, 1000 \mathrm{bar}, 120 \mathrm{~min}\right)$

6. carburize

7. finish machining (gear grinding)

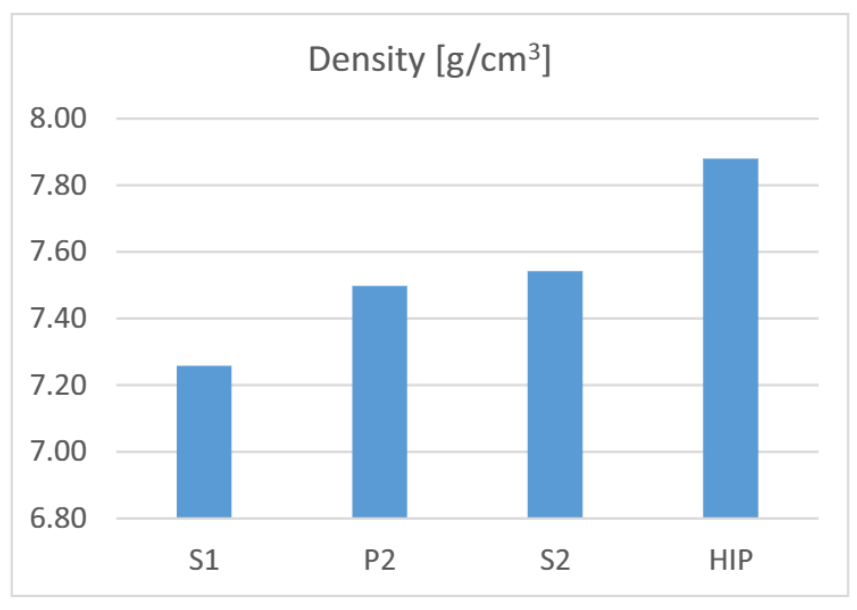

\section{FIGURE 6}

Density development after each step.

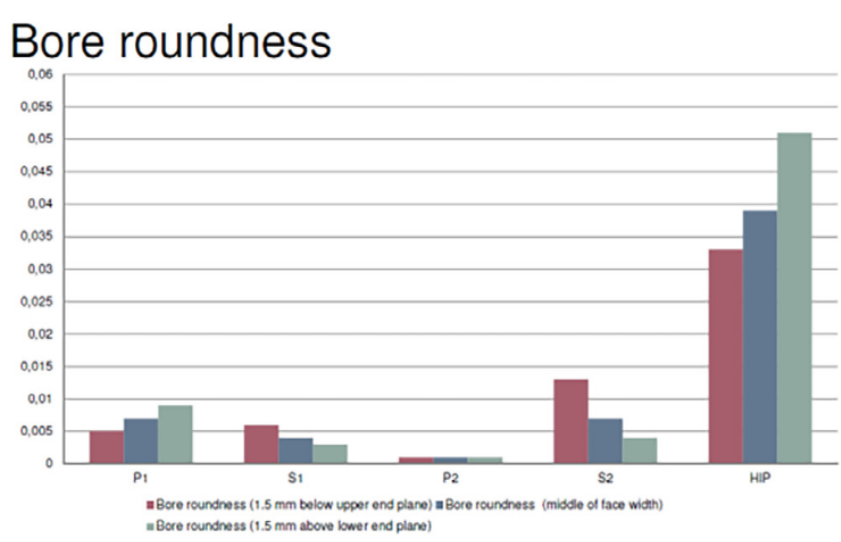

The density development after each step is presented in Figure 6 , where it can be concluded that the material has reached full density after the HIP step.

As was mentioned above there will be surface porosity remaining after the HIP process, which can have a detrimental impact on fatigue strength. On the gear flank these pores will be removed during grinding, but normally the tooth root is not ground. However, for this project, it was decided to include tooth root grinding on to ensure best possible performance.

To keep track of the dimensional changes and distortions the gears were measured after the different process steps. Especially during the second sintering and HIP:ing the components will shrink significantly and it's important to have these changes in control to ensure the final dimension of the component.

During the initial stages of the project, dimensional changes of small cylinders were used to measure the shrinkage and was also used as a basis for the tool design. In the end it was found that the HIP:ed gear was very close in size compared to the ideal value with a MOB value of $85.09 \mathrm{~mm}$ compared to the theoretical value of $85.12 \mathrm{~mm}$. This shows that even though there is a significant shrinkage it can be controlled.

Figure 7 shows the bore roundness and the run-out after the different steps. As can be seen in the figure there is an increase in the distortions after HIP:ing. However, for the demonstrator, all tolerances were within the limits of what could be corrected during the final gear grinding resulting in a gear of high quality.

\section{Fatigue testing}

The initial ambition of the project was to produce a material with higher strength than reference wrought steels, and to test the fatigue strength of the HIP:ed gears both FZG testing for contact fatigue and pulsator testing for tooth root bending fatigue were performed. The resulting SN-curves are shown in Figure 8, where it can be seen that the fatigue strength is somewhat lower than the reference material which is a typical case hardening steel, $17 \mathrm{NiCrMoS6}$. It should be noted that the fatigue testing was done before it was realized that the process caused significant grain growth, see also discussion above.

Some retests in the pulsator were also done on gear that were normalized and re-hardened to reduce the grain size. This

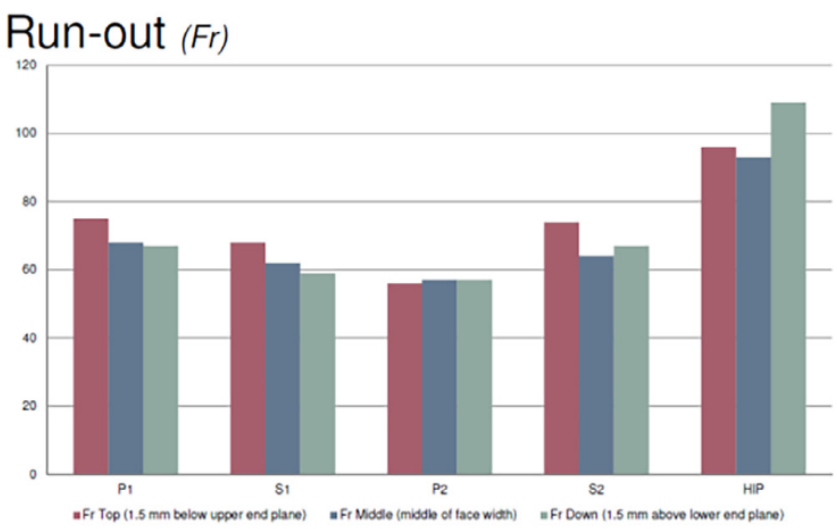



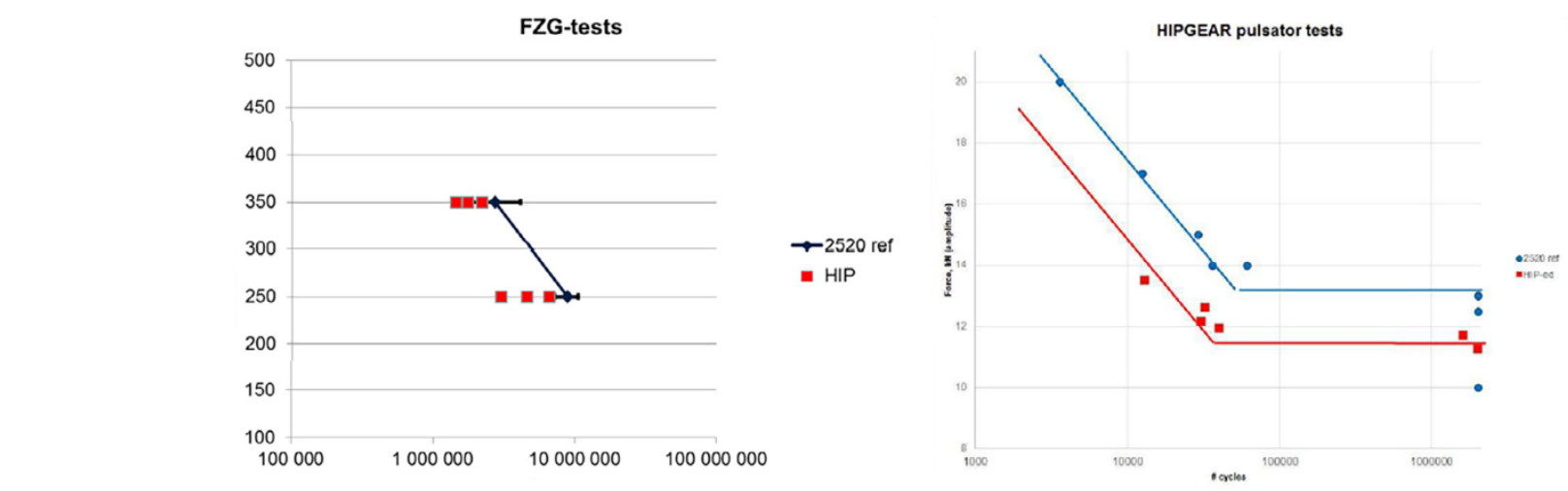

FIGURE 8

Results from fatigue testing, a. FZG testing and b. pulsator testing.

improved strength, but it was still a bit below the reference. However, the re-hardened gears showed less compressive residual stresses. This is believed to be due to the fact that they were ground before heat treatment and the beneficial effect of grinding thus removed.

\section{Summary and conclusions}

It has been shown how traditional PM manufacturing can be combined with a container less HIP:ing if the initial density is sufficiently high, thus producing a more or less pore free material. In the present study, it was decided to use double pressing to get the density up, but there are ideas of how to streamline the process by using new advanced powder mix technologies and single compaction.

In was also demonstrated how this new process can be used to make a full density powder metal gear. It was found that even though distortions increase during HIP:ing the dimensions of the component could be kept under control, to produce a gear of high quality after grinding.

A couple of challenges were also identified. For instance HIP: ing cannot close pores that are open to the surface, resulting in some remaining porosity. For the demonstrator, this was solved by gear grinding of both tooth flank and root to remove the surface layer. Furthermore, fatigue strength was found to be some- what lower than the reference material. Deeper investigation of the HIP:ed material revealed a significant grain growth, and it was concluded that this was a main contribution to the lower strength. However, it was also demonstrated how a normalization in the HIP could be used to reduce grain size to improve properties without adding additional process steps.

Finally, the project also demonstrated how simulations can be used to support process development, for instance, to calculate distortions during HIP:ing or to investigate how suitable different gear geometries are for the process.

\section{Acknowledgements}

This work was financed by the national agency VINNOVA under contract 2013-05594 within the Programme FFI. The project consisted of the following partners: Chalmers University of Technology, Höganäs AB, KTH Royal Institute of Technology, Leax AB, Quintus Technologies AB, Scania CV AB, Swepart AB, Swerea KIMAB AB, Uddeholm AB and Volvo AB.

\section{References}

[1] A. Flodin et al., Metal Powder Report 72 (2) (2017) 107-110.

[2] Sundaram M. Vattur et al., Int. J. Adv. Manuf. Technol. 99 (5-8) (2018) 17251733. 